\title{
Episodic Mass Loss and Pre-SN Circumstellar Envelopes
}

\author{
Nathan Smith \\ Astronomy Department, University of California, Berkeley, CA 94720, USA \\ email: nathans@astro.berkeley.edu
}

\begin{abstract}
I discuss observational clues concerning episodic mass-loss properties of massive stars in the time before the final supernova explosion. In particular, I will focus on the mounting evidence that LBVs and related stars are candidates for supernova progenitors, even though current paradigms place them at the end of core-H burning. Namely, conditions in the immediate circumstellar environment within a few $10^{2}$ AU of Type IIn supernovae require very high progenitor mass-loss rates. Those rates are so high that the only known stars that come close are LBVs during rare giant eruptions. I will highlight evidence from observations of some recent extraordinary supernovae suggesting that explosive or episodic mass loss (a.k.a. LBV eruptions like the 19th century eruption of Eta Car) occur in the 5-10 years immediately preceding the SN. Finally, I will discuss some implications for stellar evolution from these $\mathrm{SNe}$, the most important of which is the observational fact that the most massive stars can indeed make it to the ends of their lives with substantial $\mathrm{H}$ envelopes intact, even at Solar metallicity.
\end{abstract}

Keywords. circumstellar matter - shock waves - stars: evolution - stars: mass loss - stars: winds, outflows - supernovae: general

\section{Introduction}

Supernovae are one of the most influential ways that massive stars act as cosmic engines, energizing and polluting the ISM (especially the early ISM). To evaluate the role of massive stars as cosmic engines observationally, we must first understand the relationship between massive star evolution (mass loss, rotation, metallicity) and the type of SN observed, since SNe and can be seen to large distances. The flip side to that coin is that if we can understand the relationship between different SN properties and the star's evolution locally, then SNe also become our best probe of mass loss, circumstellar structure, stellar evolution, the initial mass function, and star formation rates throughout cosmic time. In fact, one could argue that $\mathrm{SNe}$ are the only reliable way to directly probe mass loss of individual stars at large distances where spatially resolving individual stars and separating their light from their host environment is hopeless.

One of the most important questions concerns the connection between the type of SN observed and the star's initial mass. Whether a SN is a normal Type II with $\mathrm{H}\left(8-20 \mathrm{M}_{\odot}\right.$ stars), a Type Ib/c that has shed its $\mathrm{H}$ envelope and probably died as a WR star, or a Type IIn with dense circumstellar material, is determined mainly by the star's mass-loss rate during evolution. A key prediction of most current stellar evolution models is that all stars above some mass, say $\sim 30 \mathrm{M}_{\odot}$, will fully shed their $\mathrm{H}$ envelopes and explode as WR stars, making SNe of Type Ib/c. Recent observations of SNe, on the other hand, are making it difficult to avoid the conclusion that this prediction is wrong.

Namely, SNe that have dense circumstellar material suggest to us that a small fraction of stars - probably the most massive stars — have violent mass-loss events that precede the final SN explosion. When $\mathrm{H}$ is present, these are seen as Type IIn SNe, making the most luminous SNe in the Universe. 
I should preface the discussion below by saying that exploding LBVs or LBV-like stars represent a small fraction of observed core-collapse SN events. The vast majority are normal Type II's (see Smartt, this volume), while 15-20\% are normal Type Ib/c SNe. That is perhaps not so surprising though, given that the most massive stars and LBVs themselves are quite rare.

\section{LBVs as Supernova Progenitors}

I'll start with a list of observational clues that some SNe occur when their progenitor star was in (or had recently been in) the LBV phase, contrary to expectations. Each of these on their own is compelling if not necessarily convincing, but taken together, they paint a consistent picture of LBVs as SN progenitors that is hard to dismiss.

- SN 2006jc: While this was not a Type IIn event (it was a peculiar Type Ib), it is unique so far in that it is the only SN that was actually observed to have an LBVlike outburst 2 yr before exploding as a SN. The eventual supernova showed an unusual spectrum and dust formation that was caused by very dense circumstellar material (see Foley et al. 2007; Pastorello et al. 2007; Smith et al. 2008).

- SN 2005gl: This Type IIn supernova had a progenitor star identified on preexplosion images, which was a blue/yellow supergiant that had a luminosity and colors indicative of an LBV star (Gal-Yam et al. 2007).

- Radio modulations: Kotak \& Vink (2006; and this volume) suggested that semiperiodic modulations in the radio lightcurves of a few $\mathrm{SNe}$ might be caused by the shock running into density variations caused by normal S Dor-type excursions of an LBV progenitor. The suggested cases were SNe 2001ig and 2003bg, both of which transitioned between Type Ib/c and Type II spectra (or the reverse) as they evolved, and possibly also SN 1979 C and 1998bw. However, I should note that alternative interpretations of the radio modulations have been forwarded as well (see Ryder et al. 2004; Soderberg et al. 2006; Schwarz \& Pringle 1996).

- Circumstellar Nebulae: There are three objects in our galaxy that are near twins of the unusual ring nebula around SN 1987A: HD 168625 is an LBV (Smith 2007), while SBW1 (Smith et al. 2007a) and Sher 25 (Smart et al. 2002) have abundances inconsistent with passage through a previous RSG phase. The nearly identical nebular ring structures suggest that the progenitor of SN 1987A had recently been in a similar evolutionary state when it exploded (see Smith 2007 and Smith et al. 2007a for further discussion).

- Luminous Type IIn Supernovae: The very high-luminosity of some Type IIn SNe can only be accounted for if the star had a huge mass-loss event in the decade before the SN. The clearest cases, which are also the three most luminous SNe ever observed, are SN 2006gy, SN 2005ap, and 2006tf (Smith et al. 2007b; Ofek et al. 2007; Smith \& McCray 2007; Woosley et al. 2007; Quimby et al. 2007, and Smith et al., 2008b); these require mass-loss rates on the order of $1 \mathrm{M}_{\odot} \mathrm{yr}^{-1}$ in the few years before the SN. SN 1979C, 1988Z, and others, also suggest LBV-like mass loss based on their extended highluminosity CSM interaction. In some cases, like SN2006gy and 2006tf, the progenitor's wind speed is observed in the narrow $\mathrm{P}$ Cyg $\mathrm{H} \alpha$ absorption, and its speed is $\sim 200 \mathrm{~km} \mathrm{~s}^{-1}$. That's too fast for a RSG, but just right for an LBV.

This last point is probably the most interesting, in my view, because it highlights a relatively unfamiliar phenomenon that is truly remarkable, and that I think deserves more attention. Namely, the high luminosity of these Type IIn SNe require huge bursts of episodic mass loss right before they explode. In order to produce a Type IIn spectrum, the luminosity from CSM interaction must be comparable to or larger than the luminosity from recombination of the SN ejecta or radioactive decay, which are characteristically 
about $10^{9} \mathrm{~L}_{\odot}$. A convenient expression for the progenitor's mass-loss rate needed to produce an observed luminosity $L_{9}=L_{\mathrm{SN}} /\left(10^{9} \mathrm{~L}_{\odot}\right)$ through CSM interaction, with an optimistic $100 \%$ efficiency of converting shock kinetic energy into visual light, is given by

$$
\dot{M}=0.04 L_{9} \frac{v_{w}}{200}\left(\frac{v_{\mathrm{SN}}}{4000}\right)^{-3} \mathrm{M}_{\odot} \mathrm{yr}^{-1}
$$

where $v_{w}$ and $v_{\mathrm{SN}}$ are the progenitor's wind speed and the SN blast wave speed, respectively, in $\mathrm{km} \mathrm{s}^{-1}$. L can be measured from the light curve, while $v_{w}$ and $v_{\mathrm{SN}}$ can usually be measured from the narrow and relatively broad components of the $\mathrm{H} \alpha$ line. For the main peak of SN 2006gy, the observed luminosity of $L_{9}=50$ at $70 \mathrm{~d}$ after explosion and the observed speeds of $v_{w} \simeq 200 \mathrm{~km} \mathrm{~s}^{-1}$ and $v_{\mathrm{SN}} \simeq 4,000 \mathrm{~km} \mathrm{~s}^{-1}$ required a mass-loss rate for the progenitor of $\sim 2 \mathrm{M}_{\odot} \mathrm{yr}^{-1}$ for $5-10 \mathrm{yr}$. That's the most extreme example, but its easy to see that mass-loss rates more than about $10^{-2} \mathrm{M}_{\odot} \mathrm{yr}^{-1}$ are needed in order for the CSM interaction luminosity to compete with the normal luminosity source of the SN.

If we look around us and ask "Among known stars in the Universe, which ones have the requisite mass loss to produce Type IIn SNe?", the only viable candidates with mass-loss rates above $10^{-2} \mathrm{M}_{\odot} \mathrm{yr}^{-1}$ are $L B V s$ during a giant eruption. If they are not bona-fide LBVs, then Type IIn progenitors are doing a darn good job of impersonating the $\mathrm{H}$ composition, wind speeds, and mass-loss rates of LBVs.

\section{Synchronicity}

The argument that the heavy mass loss occurs in the decade or so immediately preceding the SN is pretty straightforward. In the 100 days or so after explosion when the $\mathrm{SN}$ is bright and shows a Type IIn spectrum, it is sweeping through a radius of only a few $100 \mathrm{AU}$ (typical observed blast wave speeds are only a few $1000 \mathrm{~km} \mathrm{~s}^{-1}$, because the dense material decelerates the blast wave). In Type IIn SNe, the progenitor's wind speed can be observed in the narrow $\mathrm{P}$ Cygni absorption of $\mathrm{H} \alpha$, usually indicating speeds of a couple $100 \mathrm{~km} \mathrm{~s}^{-1}$. Thus, the radius out to which the blast wave reaches in the time it is being observed corresponds to the star's mass loss during the previous decade.

\section{Deaths of the Most Massive Stars}

Type IIn supernovae give us the most luminous SNe known in the Universe. A natural tendency is to associate them with the deaths of the most massive stars. Here are some reasons to favor that interpretation:

The classical LBVs that are expected to have giant eruptions à la Eta Carinae are the most luminous stars known, with initial masses of $60-150 \mathrm{M}_{\odot}$, although there are also some lower luminosity LBVs that are probably post-RSGs (Smith, Vink, \& de Koter 2004 ) which may arise from stars with initial masses of perhaps $25-40 \mathrm{M}_{\odot}$. Since LBV eruptions provide our only observed precedent for the required mass-loss rates of Type IIn SNe, the simplest assumption is that Type IIn's do indeed represent the rare deaths of the most massive stars.

Aside from giant LBV eruptions, the "pulsational pair instability" is the best bet going (Woosley et al. 2007), as it is the only theoretically-proposed mechanism to produce mass loss similar to LBV eruptions, and it is expected to occur right before the SN. However, the instability only occurs for initial mass above $\sim 95 \mathrm{M}_{\odot}$ (Woosley et al. 2007). Thus, if this mechanism is responsible for the pre-SN mass loss of Type IIn's, then they REALLY must be the deaths of the most massive stars. 
The brightest Type IIn SNe are energetic events, with combined luminous + kinetic energies well in excess of $10^{51}$ ergs (for example, 06gy emitted more than that in visible light alone). High-energy SN explosions are not something we associate with stars of moderate mass (i.e. $8-20 \mathrm{M}_{\odot}$ ).

(This is a bit of a tangent, but recent results show that $\sim 30 \mathrm{M}_{\odot}$ black holes exist [Prestwich et al. 2007; Silverman \& Filippenko 2008]. Those must come from the corecollapse deaths of very massive stars that did shed all their $\mathrm{H}$, because the most massive H-free WR stars are less than that [Smith \& Conti 2008].)

The luminous Type IIn's, like SN 2006gy, appear to eject 10's of $\mathrm{M}_{\odot}$ in the decade or so before the SN. Very massive stars seem to be able to do this (Smith \& Owocki 2006), but it is hard to believe that an $8-20 \mathrm{M}_{\odot}$ star could shed that much of its mass in a couple years. Furthermore, we would have no explanation for why only a small fraction of these stars have violent precursor events, whereas most die as normal Type II-P SNe. On the other hand, the most massive stars are rare compared to stars of 8-40 $\mathrm{M}_{\odot}$, so its natural that their deaths would be a small fraction of all core-collapse SNe.

Lastly, wind speeds of progenitor stars can be gleaned from the narrow $\mathrm{P}$ Cygni $\mathrm{H} \alpha$ absorption in the spectra of Type IIn SNe, and they typically have fast winds of a few $10^{2} \mathrm{~km} \mathrm{~s}^{-1}$, characteristic of LBVs (see Smith et al. 2007b). Moderately massive stars (initial mass 8-20 $\mathrm{M}_{\odot}$ ) should die as RSG's, with wind speeds of $10-20 \mathrm{~km} \mathrm{~s}^{-1}$.

Conclusion: Type IIn progenitors are NOT moderately massive stars (initial masses of 8-20 $\left.\mathrm{M}_{\odot}\right)$, but must be very massive stars, with initial masses that are probably above 50-60 $\mathrm{M}_{\odot}$. Since they die with a lot of $\mathrm{H}$, this is bad news for stellar evolution models.

\section{LBVs, LBV Impostors, SN Impostors, and SNe}

Since LBV-like eruptions are apparently responsible for the conditions that make the most luminous SNe in the Universe, our ignorance of their underlying physical mechanism is rather embarrassing.

1. One possibility is that the progenitor stars are in a regular LBV phase, that giant LBV eruptions occur repeatedly, and that some of these coincidentally occur shortly before the final SN explosion. The natural implication is that while some eruptions occur within a decade or so of the SN, many more probably will not. Statistically, this is a bit troubling, because Type IIn's represent $2-5 \%$ of core collapse SNe (Capallaro et al. 1997). If those correspond to the ones that have had giant eruption-like mass loss in the decades before the SN, then there must be at least 10 times more that are in a quiescent phase between giant eruptions. To achieve this from a normal Salpeter IMF, we would need to have all stars with initial masses above about $40 \mathrm{M}_{\odot}$ die as LBVs.

2. A second possibility is that these LBV-like eruptions really represent a precursor to the Type IIn SN, and their synchronization is not a coincidence. This could be the case if the SN-precursor mass ejections are in fact associated with an instability, perhaps the pulsational pair instability or some other instability leading to explosive mass loss in the very final nuclear burning stages. In that case, to get the observed rate of Type IIn's from a Salpeter IMF, we'd need all stars above $85-90 \mathrm{M}_{\odot}$ to explode in this way. Interestingly, this is also the range of masses that are supposed to be susceptible to the pulsational pair instability (Woosley et al. 2007).

In either case, how would we know? Can we tell the difference observationally between a classical LBV and a pulsational pair event? It is important to reiterate that regardless of the underlying physical mechanism (which is...what, again?), an observer who witnesses a brightening of several magnitudes accompanying an ejection of $0.1-10 \mathrm{M}_{\odot}$ from a massive H-rich star would classify it as a giant LBV eruption, because that's the definition of an 
LBV eruption. So, whether one wishes to call them giant LBV eruptions, SN impostors, LBV impostors, pulsational pair instability ejections, failed SNe, explosive shell burning events, mergers, or some other name, the fact remains that if seen in an external galaxy, we'd probably call it a giant LBV eruption.

\section{Massive Stars Can Die With Hydrogen}

To me, one of the most interesting questions in massive star research is whether these Type IIn SN progenitors (1) are stars that really share the same evolutionary phase as local examples of LBVs but are exploding as SNe, or instead (2) are caused by some different underlying physical mechanism that causes LBV-like mass loss right before the SN explosion. There's also (3) the possibility that some of them are genuine pair instability SNe (Smith et al. 2007b). One or more of these is right, but no matter which one it is, a firm observational fact remains that is hard to avoid and which I want to emphasize:

\section{At nearly Solar metallicity, observed supernovae tell us that very massive stars can make it to the ends of their lives and explode with massive $\mathbf{H}$ envelopes still intact.}

This is a very important clue to understanding the evolution of massive stars, because it is in direct conflict with the predictions of stellar evolution models. One might conjecture that the predictions of stellar evolution models, which depend primarily on the adopted mass-loss rates, are wrong because they have assumed mass-loss rates that are too high....and we know they are too high. If this is true, then the current paradigm that LBVs represent only a very brief transition phase between the end of core-H burning and the beginning of core-He burning lasting a few $10^{4} \mathrm{yr}$ - is probably wrong as well, and the idea that LBVs can explode as SNe becomes more compelling.

In order to make the normal LBV phase last until core collapse, the LBV lifetime must be longer than current estimates of a few $10^{4} \mathrm{yr}-$ more like a few $10^{5} \mathrm{yr}$ - because it must outlast core He burning. The main justification for a short LBV lifetime is that there are too few of them, so statistically, their lifetimes can't be too long.

Is it possible that the time during which an evolved massive star can potentially be an LBV is actually longer? Suppose, for the sake of argument, that the specific eruptive instability that leads us to call something an LBV actually represents an intermittent, possibly recurring active phase within a much longer blue supergiant/LBV phase. In other words, suppose LBV stars go through dormant phases, like volcanoes, which last much longer than their eruptive phases. What are the consequences of that? There should be many more H-rich evolved blue supergiants that are not caught at the right moment when they show wild variability, but may or may not have observable circumstellar material. This is, of course, known to be the case. There are many blue supergiants - often called LBV "candidates" because of their spectral similarity to LBVs - which do not exhibit the specific variability that earns them bona-fide LBV status. What are these stars, if not evolved massive stars that are potential dormant LBVs? Massey et al. (2007) argued a similar point, noting that there are several hundred LBV candidates in M31 and M33, compared to 8 known from their variability. I would argue that this means the "greater LBV phase" is in fact much longer than the very rapid transition from core-H to core-He burning that we normally hear quoted. If true, it would no longer be surprising to see LBVs exploding as SNe. 


\section{Addendum: Binaries, Binaries, Binaries....}

At this point, especially at this meeting, an obvious proclamation comes to mind: "Binaries are the solution!" Namely, the requisite LBV precursor event could occur in a companion star in a massive binary system instead of the exploding star; the exploding star would be a more evolved WN/WC star, so the Type Ib/c SN then expands into its companion's dense H envelope, appearing as a Type IIn SN. This would nicely resurrect stellar evolution models, because then massive stars don't need to survive until core collapse with $\mathrm{H}$ envelopes intact. Phew!

The problem is that this actually makes things much worse. Remember that the SN and LBV-like eruption need to be synchronized to within about a decade to produce a Type IIn event. What are the chances that the $L B V$ star in a $W R+L B V$ binary system would happen to have a giant $L B V$ eruption within a decade before the WR star explodes? At this meeting, J. Eldridge noted that in binary evolutionary models for massive stars, one expects that $\sim 2-5 \%$ of WR stars will have companions in the LBV phase. But here I'd emphasize that having a companion in the LBV phase is not enough for Type IIn SNe: we also need to have that companion suffer a giant eruption $\lesssim 10$ yr before the other star's SN explosion. How likely is that? If the nominal time between recurring giant LBV eruptions is $1000 \mathrm{yr}$, then there's a 1\% chance of an LBV eruption occuring within a decade of its companion's SN — but that occurs only in the $\sim 5 \%$ of massive binaries already in the WR+LBV phase - so now we are down to $0.05 \%$. This is not nearly enough. Type Ib/c SNe make up about $15 \%$ of core-collapse SNe, while Type IIn's make up about 2-5\% (Capallaro et al. 1997). Therefore, we would need about 10-25\% of the Type Ib/c SNe to explode into a companion's LBV-eruption envelope to account for Type IIn SNe, compared to the $0.05 \%$ we might expect. What about mergers? Given the compact radii of WR stars, it seems a tall order to expect that $\sim 10 \%$ of massive binaries would merge fortuitously and eject a few solar masses of $\mathrm{H}$ only a decade before the SN.

\section{References}

Cappellaro, E., Turatto, M., Tsvetkov, D. Yu., et al. 1997, A\&\&A, 322, 431

Foley, R. J., Smith, N., \& Ganeshalingam, M., al. 2007, ApJ, 657, L105

Gal-Yam, A., Leonard, D. C., Fox, D. B., et al. 2007, ApJ, 656, 372

Kotak, R. \& Vink, J. S. 2006, A\&̊A, 460, L5

Massey, P., McNeill, R. T., Olsen, K. A. G., et al. 2007, AJ, 134, 2474

Ofek, E. O., Cameron, P. B., Kasliwal, M. M., et al. 2007, ApJ, 659, L13

Pastorello, A., Mazzali, P. A., Pignata, G., et al. 2007, Nature, 447, 829

Prestwich, A. H., Kilgard, R., Crowther, P. A., et al. 2007, ApJ, 669, L21

Ryder, S. D., Sadler, E. M., Subrahmanyan, R., et al. 2004, MNRAS, 349, 1093

Schwarz, D. H. \& Pringle, J. E. 1996, MNRAS, 282, 1018

Silverman, J. M. \& Filippenko, A. V. 2008, ApJ, 678, L17

Smith, N. 2007, AJ, 133, 1034

Smith, N. \& Owocki, S. P. 2006, ApJ, 645, L45

Smith, N. \& McCray R. 2007, ApJ, 671, L17

Smith, N. \& Conti, P. S. 2008, ApJ, 679, 1467

Smith, N., Vink, J., \& de Koter, A. 2004, ApJ, 615, 475

Smith, N., Bally, J., \& Walawender, J. 2007a, AJ, 134, 846

Smith, N., Li, W., Foley, R. J., et al. 2007b, ApJ, 666, 1116

Smith, N., Foley, R. J., \& Filippenko, A. V. 2008, ApJ, 680, 568

Smith, N. et al. 2008b, ApJ, in press (astro-ph/0804.0042)

Soderberg, A., Chevalier, R. A., Kulkarni, S. R., \& Frail, D. A. 2006, ApJ, 651, 1005

Woosley, S. E., Blinnikov, S., \& Heger, A. 2007, Nature, 450, 390 


\section{Discussion}

DAVIDSON: Nathan, you mentioned the word "gonzo" and indicated that some astronomers imagine its negative! In science, gonzo is good. Astronomy has only a few dozen gonzo objects, typically with two attributes: (1) They're at extraordinarily revealing stages in their careers, and (2) are close enough to observe really well. Apart from Eta Car, you mentioned two or three of the others. Each is worth hundreds of routine objects, because they oft show where theory fails. These objects often go neglected by observers, sometimes at crucial stages.

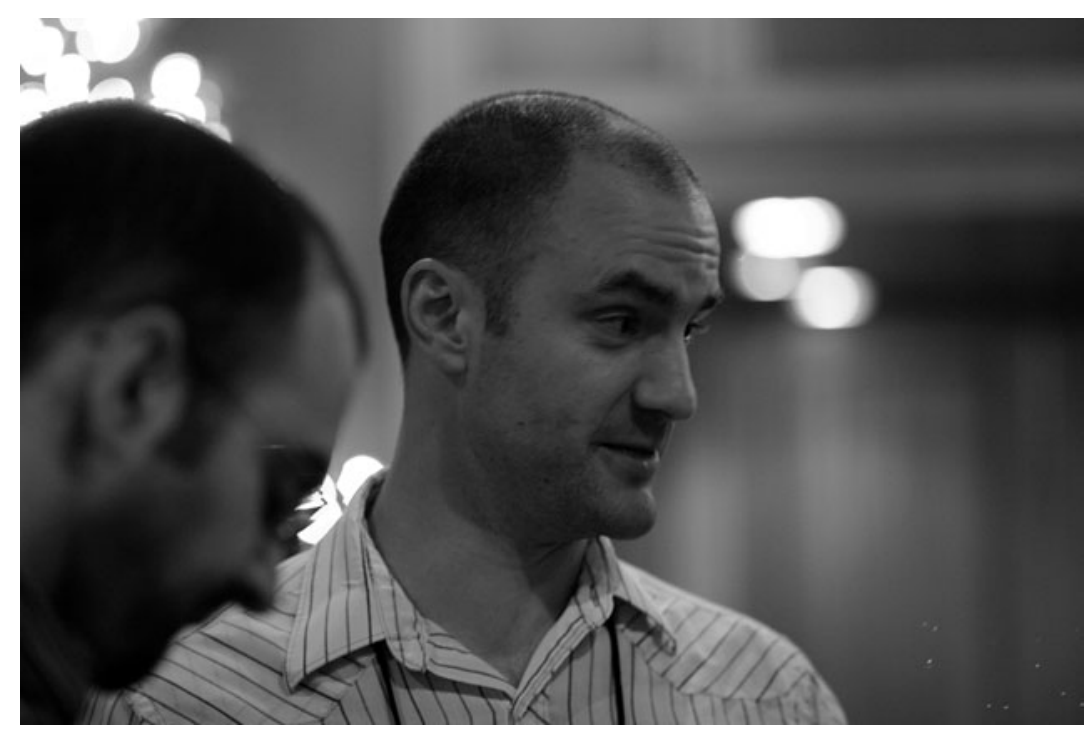

Nathan Smith.

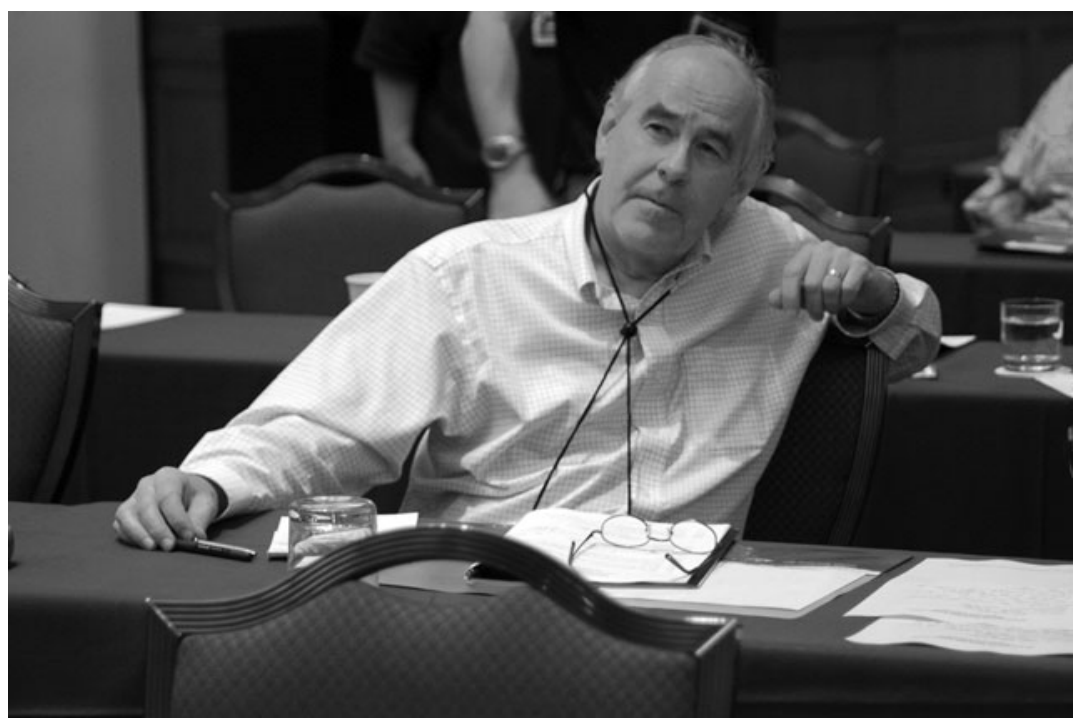

Kris Davidson. 


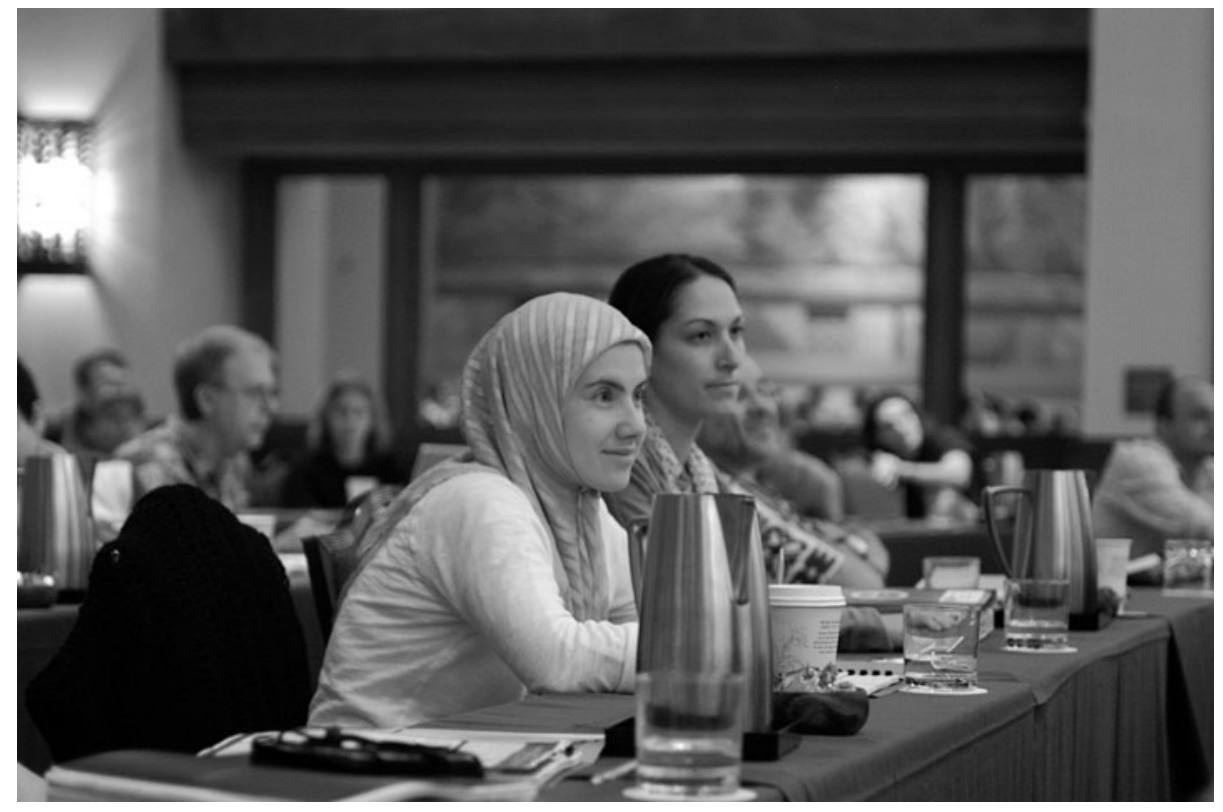

Anilmis Nurdan and Mary Oksala.

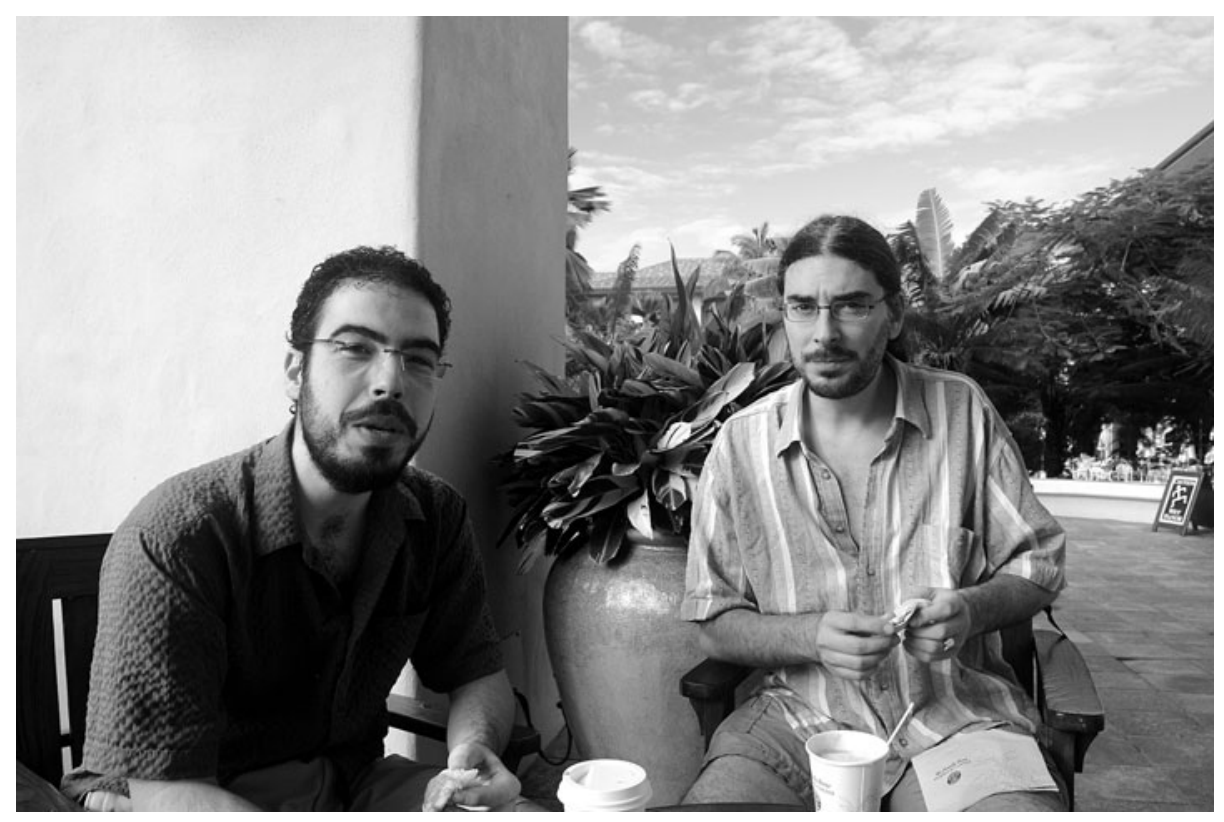

André-Nicolas Chené and Olivier Schnurr. 